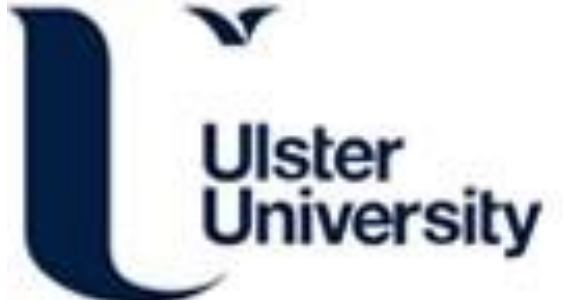

\section{Estimation of Effective Fronto-Parietal Connectivity during Motor Imagery using Partial Granger Causality Analysis}

Rathee, D., Cecotti, H., \& Prasad, G. (2016). Estimation of Effective Fronto-Parietal Connectivity during Motor Imagery using Partial Granger Causality Analysis. In Unknown Host Publication IEEE. http://uir.ulster.ac.uk/35840/2/email_ijcnn01.docx

Link to publication record in Ulster University Research Portal

\section{Published in:}

Unknown Host Publication

Publication Status:

Published online: 03/11/2016

\section{Document Version}

Author Accepted version

\section{General rights}

Copyright for the publications made accessible via Ulster University's Research Portal is retained by the author(s) and / or other copyright owners and it is a condition of accessing these publications that users recognise and abide by the legal requirements associated with these rights.

\section{Take down policy}

The Research Portal is Ulster University's institutional repository that provides access to Ulster's research outputs. Every effort has been made to ensure that content in the Research Portal does not infringe any person's rights, or applicable UK laws. If you discover content in the Research Portal that you believe breaches copyright or violates any law, please contact pure-support@ulster.ac.uk. 


\title{
Estimation of Effective Fronto-Parietal Connectivity during Motor Imagery using Partial Granger Causality Analysis
}

\author{
Dheeraj Rathee, Hubert Cecotti, Girijesh Prasad \\ Intelligent Systems Research Centre \\ School of Computing and Intelligent Systems \\ Ulster University \\ Derry/Londonderry, Northern Ireland, UK \\ email: rathee-d@email.ulster.ac.uk
}

\begin{abstract}
Connectivity analysis has become an essential tool for the evaluation of functional brain dynamics. The functional connectivity between different parts of the brain, or between different sensors, is assumed to provide key information for the discrimination of brain responses. In this study, we propose an estimation of effective cortical connectivity measures in frontal and parietal areas of human brain during four different Motor Imagery (MI) tasks. Feedback based brain-computer interface (BCI) technology has been successfully implemented for recovery of stroke patients as it can enhance the neural plasticity in brain areas associated with motor execution. However, it is still challenging to obtain reliable information regarding improvement in neural functioning during rehabilitation and its neuro-physiological dynamics. Brain connectivity is a reliable biomarker associated with brain functionality. Here, we evaluate to what extent partial granger causality can provide information in form of effective neural connectivity that can differentiate motor imagery tasks. Our results on nine subjects using the EEG dataset (BCI competition 2008 dataset 2A) show distinct connectivity patterns for all four MI classes, and higher information flow in the fronto-parietal network during task phase as compared to non-task phase. The results support the conclusion that effective connectivity analysis through partial granger causality can provide key information about neural interactions specific to different MI tasks. Moreover these interactions can be utilized as reliable biomarkers for assessment of motor recovery during stroke rehabilitation.
\end{abstract}

\section{INTRODUCTION}

Motor Imagery (MI), which involves imagination of a particular motor action without its actual execution, has showed its promising effectiveness in various research fields including sport science, neuroscience and rehabilitation. MI based brain-computer interface (BCI) systems have been studied extensively, as specific patterns of brain activity in electroencephalography (EEG) signals can be generated by various imagery tasks. This approach has been used for a wide variety of communication and control purposes, such as controlling a cursor, wheelchair or prosthesis, BCI based spellers and navigation through the virtual environment. However, recent studies have shown that MI-based BCIs can induce neural plasticity [1], and hence serve as important tools to enhance motor rehabilitation for stoke patients.
Stroke is a clinical condition wherein one or few sections of the brain lose their functionality possibly due to affected blood supply to these parts. It may lead to severe disabilities and can affect the daily life of patients, thus degrading their quality of life. In the light of our elderly societies, the encumbrance of stroke related issues is expected to grow in the future, and a crucial need to enrich our understanding of the MI related neurobiological factors emerges. Monitoring accurate neural correlates of motor activity and their dynamics during various tasks could enhance the therapeutic effects of rehabilitation procedures [2]. Functional neuroimaging techniques provide a reliable non-invasive method for deep exploration of neural mechanisms underlying reorganisation of brain networks and their effect on stroke patients recovery. BCI based rehabilitation of stroke patients has been successfully achieved in several cases although it is still difficult to analyze the direct effects of MI based BCIs on neural connectivity and recovery of the patient from stroke. Recent studies showed that brain connectivity analysis is a strong measure of cortical variations and plasticity after stroke, and it can be a useful measure for monitoring recovery of stroke patients [3].

The human brain has been divided into several areas based on their anatomical and physiological characteristics. These areas are connected to each other to form functional brain networks which are dynamically employed to perform various sensorimotor and cognitive tasks. Analyzing these network connectivities and their dynamics during various brain states may provide a better understanding of pathophysiological mechanisms related to them. However, functional connectivity evaluations are unable to provide exact information regarding the directionality of the interaction i.e. whether information flow is from area $A$ to area $B$ or vice versa. Effective connectivity analysis can derive better relationships between two areas of interest by providing causality information. Effective connectivity is therefore a strong measure for better assessment of the induced physiological variations in the brain during MI tasks.

To estimate the causal interactions between distinct brain areas, several imaging modalities can be exploited such 
as positron emission tomography (PET), functional MRI (fMTI), Magnetoencephalography (MEG) and EEG. Due to its high temporal resolution, ease of implementation and low cost, EEG has been most preferred among BCI researchers. Thus, the extraction of causality information from EEG signals can be of high significance for the advancement of MI based BCI systems for rehabilitation. Several techniques have been proposed for efficient assessment of directional interactions from EEG/MEG signals [4]. Among these methods, multivariate autoregressive (MVAR) model based methods have been widely applied to human neurophysiological signals [5], [6], [7]. In general, an MVAR based process utilizes linear difference equations to model the causal interactions between various EEG channels. It provides information about direct and indirect influences between channels representing the direction of information flow [8]. The notion of Granger causality (GC) [9] based on MVAR model, has been extensively employed to investigate directional influences within coupled variables of dynamical systems in various areas, such as climate studies [10], [11], economics [12], [13] and neuroscience [14], [15]. If prediction of any time-varying process $X$ can be enhanced by considering the past information of another time-varying process $Y$ instead of the past information of process $X$ alone, then the process $Y$ is said to granger cause process $X$. To describe the interactions between timevarying processes, three distinct frameworks of time-domain GC (bivariate, conditional and partial) have been developed in recent years [16], [17]. Fig. 1 depicts the schemes of these $\mathrm{GC}$ approaches wherein Bivariate-GC analysis is a basic technique to show causality between two concurrent coupled sources (e.g., $X(t)$ and $Y(t)$ ), conditional-GC (CGC) deal with the bipolar interactions mediated by a third source $Z(t)$ [18], and partial-GC (PGC), an extended form of CGC, considers the confounding effects of exogenous input $E$ and latent variables $L$ also [19]. PGC method enhances the efficiency of standard GC measure by mitigating the effect of confounding factors using a concept similar to partial correlation. It has been successfully implemented for performing causal connectivity analysis during multi-trial ERPs [20], [21].

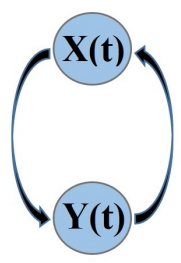

(a)

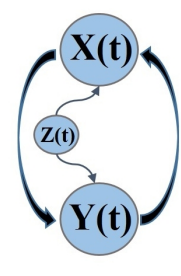

(b)

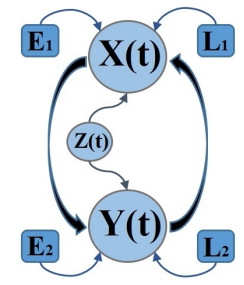

(c)
Fig. 1. Schematic diagram of (a) BGC, (b) CGC and (c) PGC.

In the present study, we estimate the effective connectivity in the fronto-parietal sensors by performing a time-domain PGC analysis of scalp EEG data involving MI tasks. In our investigation, we utilize data from five scalp electrodes including frontal $(\mathrm{Fz})$, parietal $(\mathrm{Pz})$ and sensorimotor area
(SMA) of brain. To cover SMA area, we included channel $\mathrm{C} 3, \mathrm{Cz}$ and $\mathrm{C} 4$. Our results show that (1) there exist significant changes in the effective connectivity between these areas during distinct MI tasks, and (2) it is possible to find a difference of connectivity between different motor imagery tasks. The remainder of the paper is organized as follows. First, the methods and the evaluation procedure are described in Section II. Then, the results are presented in Section III, and finally discussed in Section IV.

\section{Methods}

\section{A. Multivariate Autoregressive (MVAR) Model}

An MVAR model for a set of $L$ observed time-sampled series $x(t) \in \mathbb{R}^{L}$, with $1 \leq t \leq N, N$ is the total number of samples, and model order $r$, can be defined as follows [22]:

$$
\left(\begin{array}{c}
x_{1}(n) \\
\vdots \\
x_{L}(n)
\end{array}\right)=\sum_{p=1}^{r} A^{p}\left(\begin{array}{c}
x_{1}(n-p) \\
\vdots \\
x_{L}(n-p)
\end{array}\right)+\left(\begin{array}{c}
q_{1}(n) \\
\vdots \\
q_{L}(n)
\end{array}\right)
$$

where $\mathbf{q}=\left[q_{1}, \ldots, q_{L}\right]^{T}$ is a zero-mean white noise vector with normally distributed real-values. The auto-regression coefficient matrices $A^{p}$ are given by:

$$
A^{p}=\left(\begin{array}{ccc}
a_{1,1}^{p} & \cdots & a_{1, L}^{p} \\
\vdots & \ddots & \vdots \\
a_{L, 1}^{p} & \cdots & a_{L, L}^{p}
\end{array}\right)
$$

where $1 \leq p \leq r$. The matrix $A^{p} \in \mathbb{R}^{L \times L}$ reveals the linear interactions between any two series at the time delay $p$. For a reliable estimation using MVAR modeling, the total number of available data points $(L N)$ must be significantly higher than the total number of estimated parameters $\left(L^{2} r\right)$ [22].

\section{B. Time-domain Partial Granger Causality Analysis}

Time-domain Partial Granger Causality (PGC) is a robust form of granger causality wherein causal interactions between multivariate data can be analyzed using MVAR modeling. Unlike bivariate GC and conditional GC, it provides better estimation of the true interactions by mitigating the effect of confounding variables[19].

Let's assume three time series data including $X(t), Y(t)$ and $Z(t)$. Now to analyze the effective connectivity between $X(t)$ and $Y(t)$ (conditioned on $Z(t)$ ) based on PGC rules, the reduced model (inclusion of past values of the sink variable conditioned on other variables) can be defined by:

$$
\begin{array}{r}
X(t)=\sum_{p=1}^{k}\left(a_{1, p} X(t-p)\right)+\sum_{p=1}^{k}\left(c_{1, p} Z(t-p)\right)+ \\
\epsilon_{1}(t)+\epsilon_{1}^{E}(t)+\beta_{1}(L) \epsilon_{1}^{L}(t) \\
Y(t)=\sum_{p=1}^{k}\left(b_{1, p} X(t-p)\right)+\sum_{p=1}^{k}\left(d_{1, p} Z(t-p)\right)+ \\
\epsilon_{2}(t)+\epsilon_{2}^{E}(t)+\beta_{2}(L) \epsilon_{2}^{L}(t)
\end{array}
$$

where $p$ is the model order, $\epsilon(t)$ is the prediction error, $\epsilon^{E}(t)$ and $\beta(L) \epsilon^{L}(t)$ are the residual errors corresponding to 
exogenous (E) and latent (L) inputs, respectively. Similarly, the full model (inclusion of past values of the sink variable along with past values of source variable conditioned on rest of variables) can also be defined as:

$$
\begin{gathered}
X(t)=\sum_{p=1}^{k}\left(a_{2, p} X(t-p)\right)+\sum_{p=1}^{k}\left(b_{2, p} Y(t-p)\right)+ \\
\sum_{p=1}^{k}\left(c_{2, p} Z(t-p)\right)+\epsilon_{3}(t)+\epsilon_{3}^{E}(t)+\beta_{3}(L) \epsilon_{3}^{L}(t) \\
Y(t)=\sum_{p=1}^{k}\left(d_{2, p} X(t-p)\right)+\sum_{p=1}^{k}\left(e_{2, p} Y(t-p)\right)+ \\
\sum_{p=1}^{k}\left(f_{2, p} Z(t-p)\right)+\epsilon_{4}(t)+\epsilon_{4}^{E}(t)+\beta_{4}(L) \epsilon_{4}^{L}(t)
\end{gathered}
$$

The collective prediction errors can be taken from the previous equations, and are represented as:

$$
\mu_{i}=\epsilon_{i}(t)+\epsilon_{i}^{E}(t)+\beta_{i}(L) \epsilon_{i}^{L}(t)
$$

with $1 \leq i \leq 4$.

The covariance matrix for the reduced model can be generated as:

$$
R=\left[\begin{array}{cc}
\operatorname{var}\left(\mu_{1}(t)\right) & \operatorname{cov}\left(\mu_{1}(t), \mu_{2}(t)\right) \\
\operatorname{cov}\left(\mu_{2}(t), \mu_{1}(t)\right) & \operatorname{var}\left(\mu_{2}(t)\right)
\end{array}\right]
$$

Likewise, the covariance matrix for the full model as:

$$
L=\left[\begin{array}{cc}
\operatorname{var}\left(\mu_{3}(t)\right) & \operatorname{cov}\left(\mu_{3}(t), \mu_{4}(t)\right) \\
\operatorname{cov}\left(\mu_{4}(t), \mu_{3}(t)\right) & \operatorname{var}\left(\mu_{4}(t)\right)
\end{array}\right]
$$

The PGC indices can be calculated by taking the log ratio of partial variance of prediction error of reduced model and partial variance of prediction error of full model. The following two equations provide the PGC indices for $Y(t)$ causing $X(t)$ and vice-versa, respectively:

$$
\begin{aligned}
\mathcal{G}_{Y \rightarrow X \mid Z} & =\ln \left(\frac{R_{1,1}-R_{1,2} R_{2,2}^{-1} R_{2,1}}{L_{1,1}-L_{1,2} L_{2,2}^{-1} L_{2,1}}\right) \\
\mathcal{G}_{X \rightarrow Y \mid Z} & =\ln \left(\frac{R_{2,2}-R_{2,1} R_{1,1}^{-1} R_{1,2}}{L_{2,2}-L_{2,1} L_{1,1}^{-1} L_{1,2}}\right)
\end{aligned}
$$

\section{Dataset Overview}

The BCI Competition IV dataset 2A [23] has been analyzed for investigating causal interactions between various brain areas of interest. The dataset comprised of EEG signals acquired from nine subjects that were recorded using a cuebased paradigm during two sessions on different days [23]. The MI tasks include four different classes: left hand MI (class 1), right hand MI (class 2), both feet MI (class 3), and tongue MI (class 4). Each data acquisition session consists of 6 runs where each run comprised of 48 trials (12 trials for each class). Thus the complete study involved 288 trials from each session of the dataset. The data were acquired from 25 channels (22 EEG channels along with three monopolar EOG channels) with sampling frequency of $250 \mathrm{~Hz}$ and bandpass filtered between $0.5 \mathrm{~Hz}$ to $100 \mathrm{~Hz}$. Out of $22 \mathrm{EEG}$ channels, 5 channels $(\mathrm{Fz}, \mathrm{Cz}, \mathrm{Pz}, \mathrm{C} 3$ and $\mathrm{C} 4)$ are selected to study the fronto-parietal network during MI activities.

\section{Data Processing and Analysis}

Multi-trial PGC analysis involves a high computational cost. To reduce the processing time, the EEG data were downsampled from $250 \mathrm{~Hz}$ to $125 \mathrm{~Hz}$, hence reducing the trial length by half. Data belonging to MI task phase (300 to $600 \mathrm{~ms}$ ) for all trials were extracted and concatenated (all four classes separately) for session-wise inter-class analysis. For MI versus Non-MI analysis, class-wise data from MI task phase (300 to $600 \mathrm{~ms}$ ) and non-MI task phase (0 to 300 $\mathrm{ms}$ ) were considered. To counter the issues related to intertrial variations and non-stationarity, processes of detrending and demeaning of the data were performed wherein the ensemble average was subtracted from each trial separately along with division of each trial by ensemble standard deviation [24]. The coefficients of MVAR model for multitrial data were estimated using the LWR algorithm [25]. Akaike information criterion (AIC) [26] and the Bayesian information criterion (BIC) [27] techniques were used for estimating the optimal value of the model order $p$ (i.e. the number of time-lags). The expressions for these two methods are given as follows:

$$
\begin{aligned}
& A I C(p)=\log [\operatorname{det}(\Sigma)]+\frac{2 p L^{2}}{N} \\
& B I C(p)=\log [\operatorname{det}(\Sigma)]+\log (N) \frac{p L^{2}}{N}
\end{aligned}
$$

where $\Sigma$ is the estimated noise covariance matrix, $L$ is the number of EEG channels, and $N$ is the number of data samples. The model orders $p$ were calculated for each estimation in the range of $1 \leq p \leq 40$. The final model order was selected by comparing and choosing the highest model order value provided by AIC and BIC. We implemented two different techniques to confirm the legitimacy of applied regression models. Durbin-Watson whiteness test [28] has been used for approximating whiteness of uncorrelated residuals. The test returns a significant value of $\mathrm{d} \approx 1.8$, a confirmatory indication for rejection of null hypothesis. Further validation of the model was confirmed using the Ding method [24] by checking the consistency of the correlation structure. The Ding consistency test provided a higher value (nearly equal to 1), which shows that the selected MVAR model has effectively predicted the time series. Finally, to eliminate the statistical biasness the permutation resampling test was used with the values of bwin (window size of samples) and nperm (the number of permutation) as 75 and 5, respectively. The computational work has been performed on a Intel Core i7-4790 with 16 gb of memory, using MATLAB (V8.1) and the GCCA (V2.9) toolbox [29] for the estimation of causal effects.

\section{RESULTS}

The estimation of effective connectivity between the selected five channels has been conducted over two types of comparisons: pair-wise Inter-class analysis, and MotorImagery (MI) versus non-MI (n-MI) analysis. This study provides information about the cortical networks during MI state and resting state along with further analysis, and 
comparative evaluations of connectivity during distinct MI tasks (four classes). Fig. 2 includes a montage of examined scalp electrodes, showing strong effective connectivity (PGC value greater than 0.13 ) between them. For the pairwise comparisons, we consider Wilcoxon signed rank test with a false discovery rate correction for multiple comparisons across the 20 possible connectivity measures $(\mathrm{p}<0.025)$.

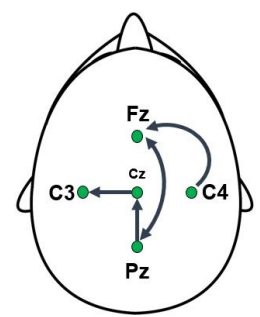

(a) Left Imagery

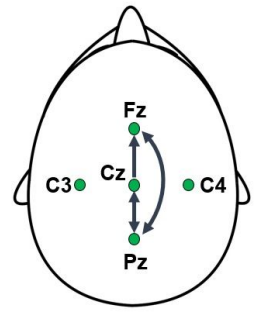

(c) Feet Imagery

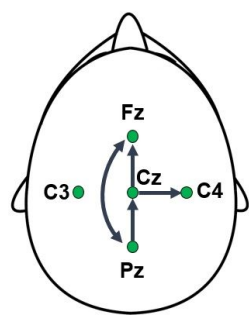

(b) Right Imagery

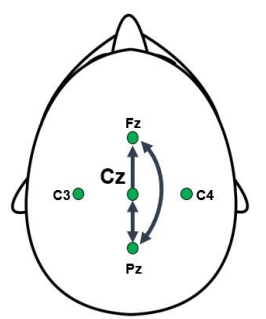

(d) Tongue Imagery
Fig. 2. Causal interactions on the EEG scalp topographical placements of electrodes corresponding to PGC values greater than 0.13 of (a) Left MI, (b) Right MI, (c) Feet MI and (d) Tongue MI.

\section{A. Inter-class Analysis}

In this part of analysis, the effective connectivity in the frontal and parietal areas of the brain with special emphasis on sensorimotor area has been estimated for each class by analyzing the EEG data related to various MI tasks. For each class and each subject, a PGC index matrix has been estimated wherein we get 20 non-zero connectivity measures. Fig. 3 shows PGC matrices for each subject during left, right, feet and tongue imagery tasks.

Furthermore, the mean values and standard deviations of 20 connectivity features across 9 subjects have been calculated for each class and a comparative plot for interclass analysis has been provided in Fig. 4. Connectivity measures which rejected the null hypothesis $(\mathrm{p}<0.05$, FDR corrected) during pair-wise inter-class comparative analysis are provided in Table I. The information from the plot and table illustrates that during right $\mathrm{MI}$, there is a higher amount of information flow from $\mathrm{C} 3$ to $\mathrm{Fz}, \mathrm{Cz}$ to $\mathrm{C} 4$, $\mathrm{C} 3$ to $\mathrm{C} 4$ and $\mathrm{C} 4$ to $\mathrm{Pz}$ whereas during left MI strong directional connectivity measures are observed from $\mathrm{C} 4$ to $\mathrm{Fz}$ and $\mathrm{C} 3$, and from $\mathrm{C} 3$ to Pz. Thus the contralateral sensorimotor area of the brain is strongly connected to the frontal area and central area while ipsilateral sensorimotor area is strongly connected to the parietal area during leftright MI tasks. The rest of the connectivity measures including $(\mathrm{Cz} \rightarrow \mathrm{Fz}),(\mathrm{Pz} \rightarrow \mathrm{Fz}),(\mathrm{Pz} \rightarrow \mathrm{C} 3),(\mathrm{Fz} \rightarrow \mathrm{Cz}),(\mathrm{C} 3 \rightarrow \mathrm{Cz})$,
$(\mathrm{C} 4 \rightarrow \mathrm{Cz}),(\mathrm{Pz} \rightarrow \mathrm{Cz}), \quad(\mathrm{Fz} \rightarrow \mathrm{C} 4),(\mathrm{Pz} \rightarrow \mathrm{C} 4), \quad(\mathrm{Fz} \rightarrow \mathrm{Pz})$, and $(\mathrm{Cz} \rightarrow \mathrm{Pz})$ do not vary significantly during these tasks.

Effective connectivity values during both feet and tongue imagery tasks infer stronger directional causality from $\mathrm{C} 3$ to $\mathrm{Fz}, \mathrm{C} 3$ to $\mathrm{Pz}, \mathrm{Cz}$ to $\mathrm{Pz}$ and $\mathrm{C} 4$ to $\mathrm{Pz}$ during both feet MI, while these effective connectivity measures remain at lower values during tongue MI. All other connectivity measures stay almost similar during both feet and tongue MI. Thus, the results show that the sensorimotor area has been strongly connected to the parietal region during feet MI task as compared to tongue MI task. There are significant variations in the connectivity measures for $(\mathrm{C} 4 \rightarrow \mathrm{Fz})$, $(\mathrm{Cz} \rightarrow \mathrm{C} 3),(\mathrm{C} 4 \rightarrow \mathrm{C} 3),(\mathrm{Cz} \rightarrow \mathrm{Pz})$ and $(\mathrm{C} 4 \rightarrow \mathrm{Pz})$ during LeftFeet MI comparative analysis. Likewise, during Left-tongue analysis, differences in effective connectivity for $(\mathrm{C} 3 \rightarrow \mathrm{Fz})$, $(\mathrm{Cz} \rightarrow \mathrm{C} 3)$, and $(\mathrm{C} 3 \rightarrow \mathrm{Pz})$ are statistically significant. The study estimated high variations can be observed in effective connectivity measures for ( $\mathrm{C} 3 \rightarrow \mathrm{C} 4),(\mathrm{Cz} \rightarrow \mathrm{C} 4),(\mathrm{C} 4 \rightarrow \mathrm{Pz})$, and $(\mathrm{C} 3 \rightarrow \mathrm{Fz}),(\mathrm{C} 3 \rightarrow \mathrm{C} 4),(\mathrm{Cz} \rightarrow \mathrm{C} 4),(\mathrm{C} 3 \rightarrow \mathrm{Pz}),(\mathrm{Cz} \rightarrow \mathrm{Fz})$ during Right-Tongue and Right-Feet $\mathrm{MI}$ analysis, respectively.

\section{B. MI versus Non-MI Analysis}

In this analysis, causality interactions between five electrodes were estimated from EEG data belonging to MI task and EEG data belonging to the non-MI phase, separately. The analysis was done over multi-trial EEG data for each subject separately to get 20 non-zero effective connectivity measures. Moreover, the difference between MI based PGC measure and its corresponding non-MI based PGC measure has been calculated for all 20 non-zero pairs and for all subjects separately. Finally, the mean values and standard deviations of 20 difference of connectivity features ( $\Delta$-PGC) across 9 subjects have been calculated for each class and comparative bar plots has been provided in Fig. 5.

Results from Fig. 5 and Table II show that there has been a significant increase in almost all connectivity values across the fronto-parietal network during performance of Left, Right tasks as compared to rest state (non-MI). Statistically significant variations in connectivity values including $(\mathrm{C} 4 \rightarrow \mathrm{Fz})$, $(\mathrm{Cz} \rightarrow \mathrm{C} 3),(\mathrm{C} 4 \rightarrow \mathrm{C} 3),(\mathrm{Fz} \rightarrow \mathrm{Cz}),(\mathrm{C} 3 \rightarrow \mathrm{Cz}),(\mathrm{Cz} \rightarrow \mathrm{Pz})$ and $(\mathrm{C} 4 \rightarrow \mathrm{Pz})$ are observed during Feet $\mathrm{MI}$ analysis. Although there are not much variations in the connectivity values during tongue MI task when compared to non-MI connectivity values. The strongest differences ( $\mathrm{p}<0.01$, FDR corrected) of connectivity measures during MI versus non-MI comparative analysis for each class are provided in Table II.

\section{Discussion}

Conventionally, patient care and recovery from stroke often rely on behavioral assessments for making crucial decisions related to therapeutic procedures. Recently the whole paradigm has started shifting to biomarkers as they involve objective monitoring. Evidences suggest that variations of effective cortical connectivity can be utilized as biomarkers for stroke patient rehabilitation. MI based BCI technology has been used for rehabilitation after stroke by involving 


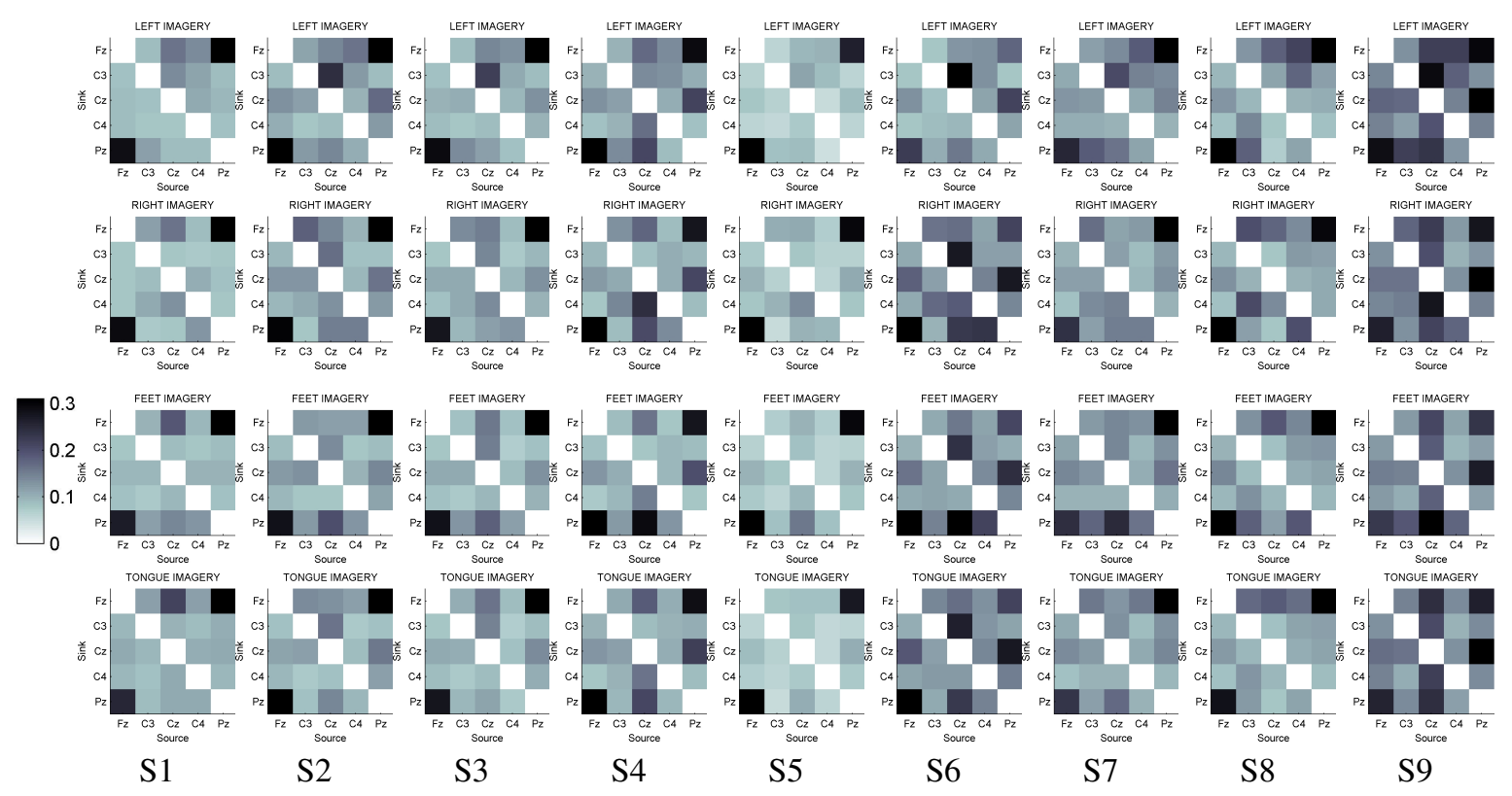

Fig. 3. Individual connectivity matrices for each subject and each MI task.

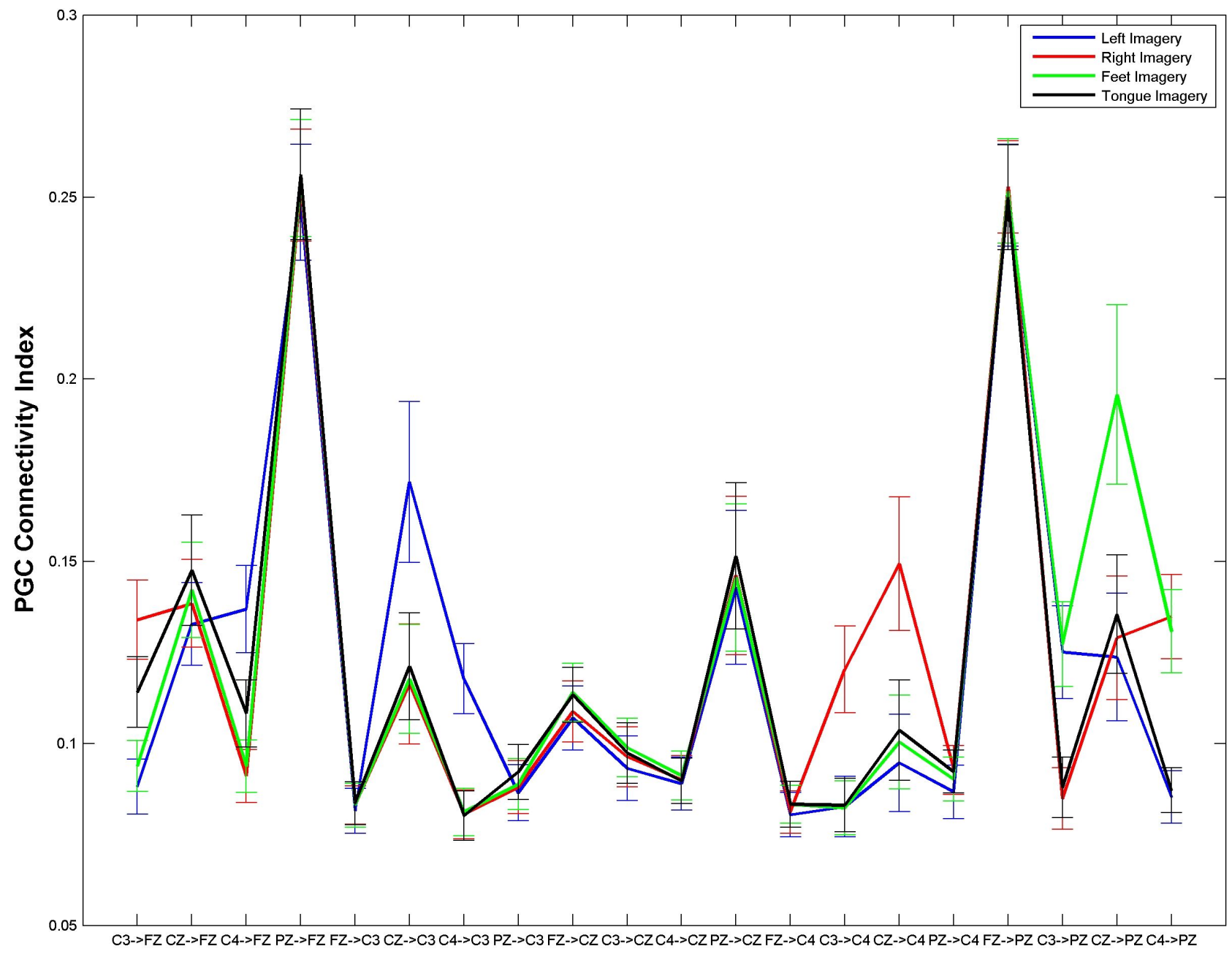

Fig. 4. PGC measures during MI tasks with pairwise comparisons. The error bar represents the standard error across subjects. 


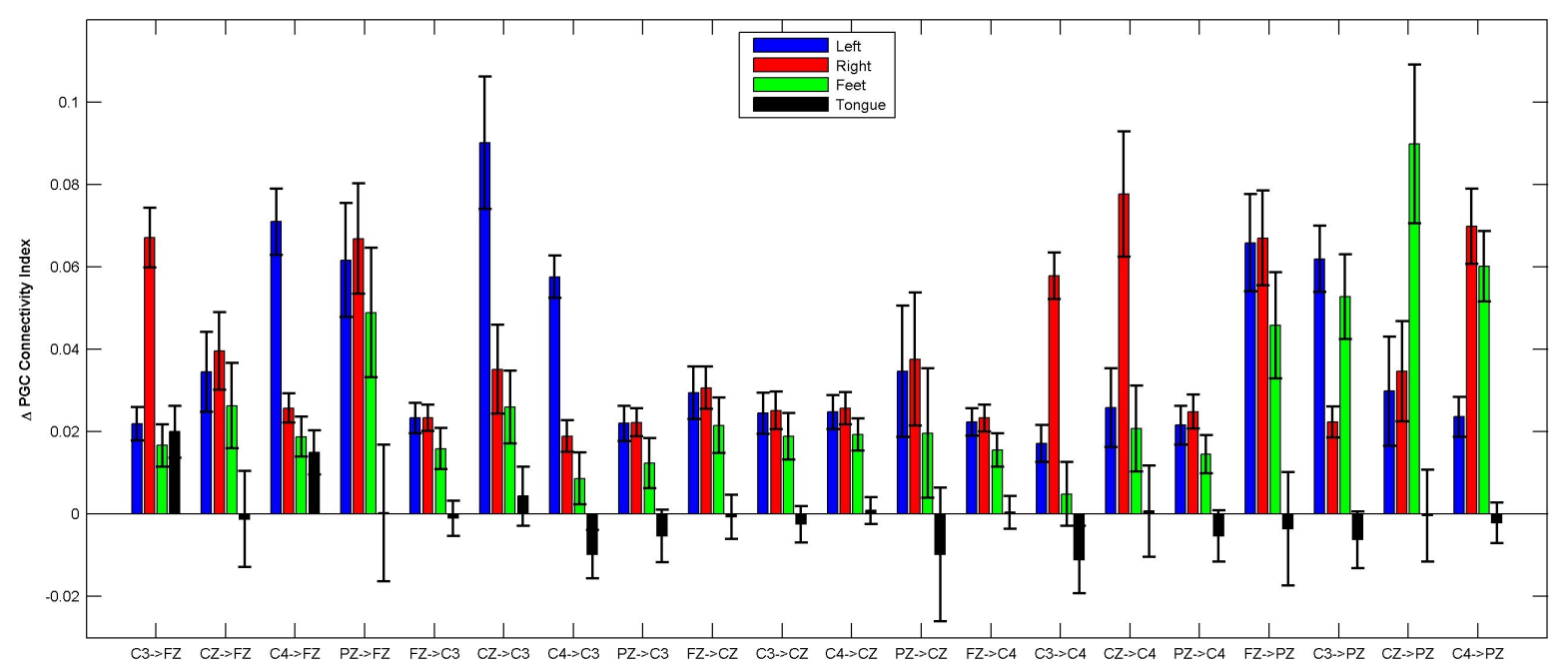

Fig. 5. Difference of PGC measures ( $\triangle \mathrm{PGC}$ ) during MI tasks with pairwise comparisons. The error bar represents the standard error across subjects.

TABLE I

SIGNIFICANT CONNECTIVITIES FOR INTER-CLASS COMPARISONS ( $<<0.05$, FDR CORRECTED).

\begin{tabular}{|c|c|}
\hline Condition & Relevant connectivities \\
\hline Left-Right & $(\mathrm{C} 3 \rightarrow \mathrm{Fz}),(\mathrm{C} 4 \rightarrow \mathrm{Fz}),(\mathrm{Cz} \rightarrow \mathrm{C} 3),(\mathrm{C} 4 \rightarrow \mathrm{C} 3),(\mathrm{C} 3 \rightarrow \mathrm{C} 4),(\mathrm{Cz} \rightarrow \mathrm{C} 4),(\mathrm{C} 3 \rightarrow \mathrm{Pz}),(\mathrm{C} 4 \rightarrow \mathrm{Pz})$ \\
\hline Left-Feet & $(\mathrm{C} 4 \rightarrow \mathrm{Fz}),(\mathrm{Cz} \rightarrow \mathrm{C} 3),(\mathrm{C} 4 \rightarrow \mathrm{C} 3),(\mathrm{Fz} \rightarrow \mathrm{Cz}),(\mathrm{Cz} \rightarrow \mathrm{Pz}),(\mathrm{C} 4 \rightarrow \mathrm{Pz})$ \\
\hline Left-Tongue & $(\mathrm{C} 3 \rightarrow \mathrm{Fz}),(\mathrm{Cz} \rightarrow \mathrm{C} 3),(\mathrm{C} 4 \rightarrow \mathrm{C} 3),(\mathrm{Pz} \rightarrow \mathrm{C} 3),(\mathrm{C} 3 \rightarrow \mathrm{Pz})$ \\
\hline Right-Feet & $(\mathrm{C} 3 \rightarrow \mathrm{Fz}),(\mathrm{C} 3 \rightarrow \mathrm{C} 4),(\mathrm{Cz} \rightarrow \mathrm{C} 4),(\mathrm{C} 3 \rightarrow \mathrm{Pz}),(\mathrm{Cz} \rightarrow \mathrm{Pz})$ \\
\hline Right-Tongue & $(\mathrm{C} 4 \rightarrow \mathrm{Fz}),(\mathrm{C} 3 \rightarrow \mathrm{C} 4),(\mathrm{Cz} \rightarrow \mathrm{C} 4),(\mathrm{C} 4 \rightarrow \mathrm{Pz})$ \\
\hline Feet-Tongue & $(\mathrm{C} 3 \rightarrow \mathrm{Fz}),(\mathrm{C} 4 \rightarrow \mathrm{Fz}),(\mathrm{C} 3 \rightarrow \mathrm{Pz}),(\mathrm{Cz} \rightarrow \mathrm{Pz}),(\mathrm{C} 4 \rightarrow \mathrm{Pz})$ \\
\hline
\end{tabular}

TABLE II

SIGNIFICANT CONNECTIVITIES FOR MI VERSUS NON-MI COMPARISONS $(\mathrm{P}<0.01$, FDR CORRECTED).

\begin{tabular}{|c|c|}
\hline Condition & Relevant connectivities \\
\hline Left & $(\mathrm{C} 4 \rightarrow \mathrm{Fz}),(\mathrm{Fz} \rightarrow \mathrm{C} 3),(\mathrm{Cz} \rightarrow \mathrm{C} 3),(\mathrm{C} 4 \rightarrow \mathrm{C} 3),(\mathrm{Pz} \rightarrow \mathrm{C} 3),(\mathrm{Fz} \rightarrow \mathrm{Cz}),(\mathrm{C} 3 \rightarrow \mathrm{Cz}),(\mathrm{Fz} \rightarrow \mathrm{C} 4),(\mathrm{Pz} \rightarrow \mathrm{C} 4),(\mathrm{Fz} \rightarrow \mathrm{Pz}),(\mathrm{C} 3 \rightarrow \mathrm{Pz})$, \\
\hline Right & $(\mathrm{C} 3 \rightarrow \mathrm{Fz}),(\mathrm{C} 4 \rightarrow \mathrm{Fz}),(\mathrm{Fz} \rightarrow \mathrm{C} 3),(\mathrm{Cz} \rightarrow \mathrm{C} 3),(\mathrm{C} 4 \rightarrow \mathrm{C} 3),(\mathrm{Pz} \rightarrow \mathrm{C} 3),(\mathrm{Fz} \rightarrow \mathrm{Cz}),(\mathrm{C} 3 \rightarrow \mathrm{CZ}),(\mathrm{C} 4 \rightarrow \mathrm{Cz}),(\mathrm{Fz} \rightarrow \mathrm{C} 4),(\mathrm{C} 3 \rightarrow \mathrm{C} 4)$, \\
& $(\mathrm{Cz} \rightarrow \mathrm{C} 4),(\mathrm{Pz} \rightarrow \mathrm{C} 4),(\mathrm{Fz} \rightarrow \mathrm{Pz}),(\mathrm{C} 3 \rightarrow \mathrm{Pz}),(\mathrm{C} 4 \rightarrow \mathrm{Pz})$ \\
\hline Feet & $(\mathrm{C} 4 \rightarrow \mathrm{Fz}),(\mathrm{Cz} \rightarrow \mathrm{C} 3),(\mathrm{C} 4 \rightarrow \mathrm{C} 3),(\mathrm{Fz} \rightarrow \mathrm{Cz}),(\mathrm{C} 3 \rightarrow \mathrm{Cz}),(\mathrm{Cz} \rightarrow \mathrm{Pz}),(\mathrm{C} 4 \rightarrow \mathrm{Pz})$ \\
\hline Tongue & $\emptyset$ \\
\hline
\end{tabular}

patients in BCI-feedback training. To improve this BCIfeedback training therapy, brain connectivity measures can be utilized for objective assessment of patient recovery. It is therefore important to study these variations in healthy patients during various motor imagery tasks, so as to provide a reliable standard for comparative diagnosis of the cortical connectivity measures of stroke patients. However, in general brain connectivity has been determined on the source and/or sensor level using fMRI, PET and MEG but these methods have restrictions in clinical applications. Moreover, high computational load and less cost-effectiveness hinder their prospective use in continuous monitoring. The current study focused on estimation of effective connectivity in frontal and parietal cortex of brain using scalp EEG.

Our results displayed a strong forward and backward effective connectivity loop between the parietal and the frontal area of brain during execution of motor imagery tasks. This concurrence is consistent with earlier neurophysiological fMRI studies, which reported correlated patterns of neural activities within the frontal and parietal brain cortices [30].
Furthermore, during left and right MI tasks, the strong forward connection between contralateral SMA and frontal area, and backward connection between ipsilateral SMA and parietal cortex provide significant information about the variations within fronto-parietal network. Similar results were reported during motor imagery and motor execution tasks in recent study [31]. In addition, we also successfully estimated the effective connectivity during feet and tongue imagery tasks. These connectivity maps provide a crucial information regarding neurophysiology during MI which can be implemented as standard features for assessment of motor recovery during $\mathrm{BCI}$ based rehabilitation of stroke patients.

\section{CONClusion}

In this paper, we have used PGC analysis on scalp EEG data from a set of five electrode $(\mathrm{Fz}, \mathrm{Cz}, \mathrm{Pz}, \mathrm{C} 3$ and C4) covering the important regions of frontal, parietal and sensorimotor area of brain. The results showed significant variations in the effective connectivity during various MI tasks. During left and right imagery tasks, there is strong 
directional connectivity from contralateral sensorimotor area to the frontal area $(\mathrm{Fz})$ while the ipsilateral area is strongly connected to the parietal area $(\mathrm{Pz})$. These results support the contralateral nature of human brain functionality and extend the preliminary analysis [7], [32]. During feet MI, higher information flow from the whole sensorimotor area $(\mathrm{C} 3, \mathrm{Cz}$ and $\mathrm{C} 4)$ to the parietal area $(\mathrm{Pz})$ has been found. MI versus non-MI analysis showed enhancement in most of the connectivity measures in fronto-parietal area in all classes except tongue MI. The current study presents reliable estimations of neural measures of effective connectivity of healthy subjects correlated to left, right, feet and tongue MI tasks, and provides key information to optimize the recovery of stroke patients using MI-based BCI systems. Future work will involve correlating effective connectivity measures with actual motor execution tasks, and to look for other biomarkers associated with sensorimotor mechanism of the human brain.

\section{Acknowledgment}

D.R. is supported by Ulster University Vice Chancellor's Research scholarship (VCRS). G.P. and H.C. are supported by the Northern Ireland Functional Brain Mapping Facility project (1303/101154803), funded by InvestNI and the University of Ulster. G.P. is also supported by the UKIERI DST Thematic Partnership project "A BCI operated hand exoskeleton based neuro-rehabilitation system" (UKIERIDST-2013-14/126).

\section{REFERENCES}

[1] S. Silvoni, A. Ramos-Murguialday, M. Cavinato, C. Volpato, G. Cisotto, A. Turolla, F. Piccione, and N. Birbaumer, "Brain-computer interface in stroke: a review of progress," Clinical EEG and Neuroscience, vol. 42, no. 4, pp. 245-252, 2011.

[2] E. Burke and S. Cramer, "Biomarkers and predictors of restorative therapy effects after stroke," Current neuro. and neurosc. reports, vol. 13, no. 2, pp. 1-10, 2013

[3] J. Wu, E. B. Quinlan, L. Dodakian, A. McKenzie, N. Kathuria, R. J. Zhou, R. Augsburger, J. See, V. H. Le, R. Srinivasan, and S. C. Cramer, "Connectivity measures are robust biomarkers of cortical function and plasticity after stroke," Brain, vol. 138, pp. 2359-2369, 2015.

[4] K. Lehnertz, "Assessing directed interactions from neurophysiological signals-an overview," Physiol. Meas., vol. 32, pp. 1715-1724, 2011.

[5] L. Faes, S. Erla, and G. Nollo, "Measuring connectivity in linear multivariate processes: Definitions, interpretation, and practical analysis," Comput. Math. Methods in Med., vol. 2012, pp. 1-18, 2012.

[6] A. Omidvarnia, M. Mesbah, M. Khlif, J. O'Toole, P. Colditz, and B. Boashash, "Dataset shift detection in non-stationary environments using ewma charts," in Proc. of the Int. Conf. of the IEEE Eng. in Medicine and Biology Society (EMBC), 2011, pp. 1423-1426.

[7] S. Hu, H. Wang, J. Zhang, W. Kong, Y. Cao, and R. Kozma, "Comparison analysis: granger causality and new causality and their applications to motor imagery," IEEE Trans. on Neural Networks and Learning Systems, vol. 99, pp. 1-16, 2015.

[8] L. Astolfi, F. Cincotti, D. Mattia, F. de Vico Fallani, A. Tocci, A. Colosimo, S. Salinari, M. Marciani, W. Hesse, H. Witte, and M. Ursino, "Tracking the time-varying cortical connectivity patterns by adaptive multivariate estimators," IEEE Trans. on Biomedical Engineering, vol. 33, no. 3, pp. 902-913, 2008.

[9] C. W. J. Granger, "Investigating causal relations by econometric models and cross-spectral methods," Econometrica, vol. 37, pp. 424438, 1969.

[10] E. Kodra, S. Chatterjee, and A. Ganguly, "Exploring granger causality between global average observed time series of carbon dioxide and temperature," Theoretical and applied climatology, vol. 104, no. 3-4, pp. 325-335, 2011.
[11] D. Stern and R. Kaufmann, "Anthropogenic and natural causes of climate change," Climatic change, vol. 122, pp. 257-269, 2014.

[12] M. Md-Yusuf and H. Rahman, "The granger causality effect between the stock market and exchange rate volatility in the asean 5 countries," in Proc. of the IEEE Symp. on Business, Engineering and Industrial Applications (ISBEIA), 2012, pp. 754-759.

[13] F. De-cheng, L. Yun, and L. Hao, "The causality between china's fiscal expenditure on science and technology and industrial structure rationalization: An empirical study," in Proc. of the IEEE Conf. on Management Science \& Engineering (ICMSE), 2014, pp. 780-785.

[14] S. E. Lakhan, A. Kirchgessner, and M. Hofer, "A copula-based granger causality measure for the analysis of neural spike train data," IEEE/ACM Trans. on Computational Biology and Bioinformatics, vol. PP, pp. 1-9, 2015.

[15] C. Chuang, C. Huang, C. Lin, L. Ko, J. Chang, and J. Yang, "Mapping information flow of independent source to predict conscious level: a granger causality based brain-computer interface," in Proc. of the IEEE Symp. on Computer, Consumer and Control (IS3C), 2012, pp. 813816.

[16] K. J. Blinowska, "Review of the methods of determination of directed connectivity from multichannel data," Medical and Biological Engineering and Computing, vol. 49, pp. 521-529, 2011.

[17] B. Roelstraete and Y. Rosseel, "Does partial granger causality really eliminate the influence of exogenous inputs and latent variables?" $J$. Neurosci. Methods, vol. 206, pp. 73-77, 2012.

[18] J. F. Geweke, "Measures of conditional linear dependence and feedback between time series," J. of the American Statistical Association, vol. 79, pp. 907-915, 1984.

[19] S. Guo, A. K. Seth, K. M. Kendrick, and al., "Partial granger causality eliminating exogenous inputs and latent variables," J. Neurosci. Methods, vol. 172, no. 79-93, 2008.

[20] V. Youssofzadeh, G. Prasad, M. Naeem, and K. Wong-Lin, "Temporal information of directed causal connectivity in multi-trial ERP data using partial granger causality," Neuroinformatics, vol. 14, no. 1, pp. 99-120, 2016.

[21] V. Youssofzadeh, D. Zanotto, P. Stegall, M. Naeem, K. Wong-Lin, S. K. Agrawal, and G. Prasad, "Directed neural connectivity changes in robot-assisted gait training: A partial granger causality analysis," in Proc. of the Int. Conf. of the IEEE Eng. in Medicine and Biology Society $(E M B C), 2014$, pp. 6361-6364.

[22] M. Tangermann, K. R. Muller, A. Aertsen, N. Birbaumer, C. Braun, C. Brunner, R. Leeb, C. Mehring, K. J. Miller, G. R. Muller Putz, G. Nolte, G. Pfurtscheller, H. Preissl, G. Schalk, A. Schlogl, C. Vidaurre, S. Waldert, and B. Blankertz, "Review of the bci competition iv," Front. Neurosci., vol. 6, p. 55, Jan. 2012.

[23] H. Hytti, R. Takalo, and H. Ihalainen, "Tutorial on multivariate autoregressive modelling," J. Clin. Monit. Comput., vol. 20, pp. 101108, 2006.

[24] M. Ding, S. L. Bressler, W. Yang, and H. Liang, "Short-window spectral analysis of cortical event related potentials by adaptive multivariate autoregressive modeling: data preprocessing,model validation, and variability assessment," Biological Cybernetics, vol. 83, pp. 35-45, 2000.

[25] M. Morf, A. Vieira, and e. a. Lee, L. D., "Recursive multichannel maximum entropy spectral estimation," IEEE Trans. on Geoscience Electronics, vol. 16, pp. 85-94, 1978.

[26] H. Akaike, "A new look at the statistical model identification," IEEE Trans. on Automatic Control, vol. 19, pp. 716-723, 1974.

[27] G. Schwarz, "Estimating the dimension of a model," Annals of Statistics, vol. 6, pp. 461-464, 1978.

[28] J. Durbin and G. S. Watson, "Testing for serial correlation in least squares regression ii," Biometrika, vol. 38, pp. 159-178, 1951.

[29] A. K. Seth, "A MATLAB toolbox for granger causal connectivity analysis," J. Neurosci. Methods, vol. 186, no. 2, pp. 262-273, 2010.

[30] S. Bajaj, A. Butler, D. Drake, and M. Dhamala, "Brain effective connectivity during motor-imagery and execution following stroke and rehabilitation," NeuroImage, vol. 8, pp. 572-582, 2015.

[31] L. Xu, H. Zhang, M. Hui, Z. Long, Z. Jin, Y. Liu, and L. Yao, "Motor execution and motor imagery: a comparison of functional connectivity patterns based on graph theory," Neuroscience, vol. 261, pp. 184-194, 2014.

[32] P. Ghosh, A. Mazumder, S. Bhattacharyya, D. Tibarewala, and M. Hayashibe, "Functional connectivity analysis of motor imagery eeg signal for brain-computer interfacing application," in Proc. of the Int. Conf. of the IEEE on Neural Engineering (NER), 2015, pp. 210-213. 